\title{
Laser-driven structural modifications and diffusion phenomena of plasmonic AIN/Ag stratified films
}

\author{
E. Delli ${ }^{1}$, C. Bazioti $^{1}$, N. Pliatsikas ${ }^{1}$, N. Kalfagiannis ${ }^{2}$, G. Vourlias $^{1}$, A. Siozios ${ }^{3}$, G.P. \\ Dimitrakopoulos $^{1}$, D.C. Koutsogeorgis ${ }^{2}$, P. Patsalas, ${ }^{1, *}$ \\ ${ }^{1}$ Department of Physics, Aristotle University of Thessaloniki, Thessaloniki, GR-54124, Greece \\ ${ }^{2}$ School of Science and Technology, Nottingham Trent University, Nottingham, NG11 8NS, United \\ Kingdom \\ ${ }^{3}$ Department of Materials Science and Engineering, University of Ioannina, GR-45110 Ioannina, \\ Greece \\ *ppats@physics.auth.gr
}

ABSTRACT: Laser annealing (LA) of AlN/Ag multilayers was proven to be an effective process to control the structure and dispersion of $\mathrm{Ag}$ into the AlN resulting in intense coloration via the localized surface plasmon resonance, which is of particular importance for decorative applications. In this work we present a study of the structural changes occurring in various AlN/Ag multilayers after LA, in an effort to establish firm knowledge of the diffusion and re-nucleation mechanisms that occur during the laser process. We investigate the effect of the basic LA parameters, such as the laser wavelength (193 and $248 \mathrm{~nm})$, fluence $\left(400-700 \mathrm{~mJ} / \mathrm{cm}^{2}\right)$, pressure (1 and $\left.10 \mathrm{Bar}\right)$ and number of pulses (1 and 2) and we show that the main processes is the Ag particle enhancement close to the film surface as a result of additive outidiffusion $\mathrm{Ag}$ and the formation of nanoparticles of varying size.

KEYWORDS: Laser Annealing; Multilayers; Diffusion; Plasmonics 


\section{INTRODUCTION}

Among the various categories of materials used for coatings, nanocomposites consisting of noble metal inclusions [1-6] are of substantial importance due to their particular functionalities that originate from the nanometric dispersion of the metallic phase and offer exceptional potential to control their mechanical behavior [7-9], and open perspectives for a variety of applications such as catalysis [10-12], biocompatible surfaces [13-17], solar harvesting [18-20], and foremost plasmonics [21-29]. In particular, the ability of controlling the optical performance via the morphology of the embedded plasmonic nanoparticles (NPs) into the coating and their localized surface plasmon resonance (LSPR) offers also great potential for decorative and ophthalmic coatings [30-33]. Such control of the plasmonic performance of metal-ceramic decorative films may be achieved by laser annealing [27-30], which offers an extraordinary combination of assets with the cold character being the most distinguished among them, as it enables the processing of film grown on temperature-sensitive organic substrates [27].

Recently, we demonstrated the subsurface formation of Ag NPs with LSPR response, embedded in $\mathrm{AlN}$ and $\mathrm{Y}_{2} \mathrm{O}_{3}$ starting from non-plasmonic metal/dielectric multilayers after subjecting those to a single shot UV (193 nm) laser annealing (LA) step and studied mostly the effect of the thermal conductivity of the ceramic matrix [27]. However, the effects of most of the LA parameters (laser wavelength, fluence, ambient pressure) were not studied thoroughly and quantitatively. In the present work, we present an investigation of the effect of the basic laser annealing parameters such as the laser wavelength $(193 \mathrm{~nm}$ and $248 \mathrm{~nm})$, fluence $\left(400-700 \mathrm{~mJ} / \mathrm{cm}^{2}\right)$, number of pulses (one or two) and the ambient pressure (one or ten Bar) to the crystal structure and morphology of three sets of multilayered AlN:Ag films of varying thicknesses of the individual AlN and Ag layers, thus providing a more complete description of the laser annealing process in such multilayers. 


\section{EXPERIMENTAL}

\subsection{Film Deposition}

The multilayers investigated in this work consisted of alternate thin films of AlN and Ag. All samples were deposited on Czochralski grown, n-type Si (001) wafers. Prior to deposition, the substrates were chemically cleaned in an ultrasonic bath and subsequently purged with dry $\mathrm{N}_{2}$ gas. The growth took place in a high vacuum system (base pressure at $2 \times 10^{-6} \mathrm{~Pa}$ ) employing the Dual Cathode Reactive Magnetron Sputtering (DCRMS). Pure Al and Ag round (3") sputter targets of 99.99\% purity were used. The Ar (99.999\% pure) and $\mathrm{N}_{2}$ (99.999\% pure) flow rates were kept constant at 12 and $8 \mathrm{sccm}$, respectively, throughout the deposition (during Ag deposition as well). In order to vary the crystallinity of the AlN layers, the sputtering power applied to the $\mathrm{Al}$ cathode varied from $15-100 \mathrm{~W}$. In addition, the deposition time was varied in order to control the intermediate layer thicknesses. The deposition conditions for each sample, along with their structural features are summarized in Table 1. During deposition, the substrate holder was rotated at $12 \mathrm{rpm}$ in order to ensure the uniformity of the layers.

\subsection{Laser Annealing}

Following the growth process, the samples were subjected to LA, which is an ultra-fast processing method that allows the subsurface modification of the multilayered materials and promotes the metallic NPs' formation below the dielectric AlN surface. Two laser sources have been used in the present study: An ArF excimer laser emitting light at $193 \mathrm{~nm}$ and a $\mathrm{KrF}$ excimer laser emitting light at $248 \mathrm{~nm}$. Both laser sources are capable of providing unpolarized light pulses with duration of 25ns. A system of optics was used (described in detail elsewhere [27-30]) in order to deliver a $2.5 \times 2.5 \mathrm{~mm}^{2}$ square (top hat profile) spot to the sample. An optical attenuator was used to allow for delivering pulses of varying fluence namely $400,500,600$ and $700 \mathrm{~mJ} / \mathrm{cm}^{2}$. The samples were processed with one and two pulses. Finally, the LA process was performed both under ambient and pressurized environments. For the latter case Ar gas was introduced into a 
special designed chamber, with dedicated optical windows for each laser source, where the sample seats.

\subsection{Characterization Methods}

X-ray diffraction (XRD) was employed to provide information about the nanostructure of the films. A Rigaku Ultima ${ }^{+}$diffractometer equipped with a $\mathrm{CuK}_{\alpha}$ anode (wavelength $0.15406 \mathrm{~nm}$ ) was used. The XRD patterns from $2.5 \times 2.5 \mathrm{~mm}^{2}$ samples were recorded by grazing incidence geometry at an angle of $2^{\circ}$; the later measurements were performed in order to increase the signal to noise ratio coming from the exceptionally fine Ag NPs. The quantitative analysis was based on Lorentzian broadening of the peaks and applying Scherrer's formula [34] to estimate the grain size of Ag in the films. Although XRD lacks the resolution of transmission electron microscopy (TEM), it provides structural information of higher statistical significance all over the volume and surface of the sample. For this reason XRD was employed in order to extract quantitative structural information for the samples after LA.

Cross sectional transmission electron microscopy (XTEM) and high-resolution TEM (HRTEM) observations were performed in a $200 \mathrm{kV}$ JEOL 2011 and a $200 \mathrm{kV}$ JEOL 2000 microscopes. TEM sample preparation was performed by mechanical grinding using the sandwich technique, followed by low energy $\mathrm{Ar}^{+}$ion milling in the GATAN PIPS.

X-ray photoelectron spectra were acquired in a KRATOS Axis Ultra DLD system equipped with a monochromated $\mathrm{AlK}_{\alpha} \mathrm{X}$-Ray source, a hemispherical sector analyzer and a multichannel detector. The XPS measurements were acquired using $20 \mathrm{eV}$ pass energy resulting in a full width at half maximum of the Ag-3d peak less than $500 \mathrm{meV}$. Spectral shifts due to charging of the surface were evaluated and subtracted based on the spectral positions of the C-1s peak of adventitious Carbon $(284.6 \mathrm{eV})$. 


\section{RESULTS AND DISCUSSION}

\subsection{Structure of as grown samples}

The samples investigated in the present study varied in the AlN and Ag layer thicknesses and in the crystallinity of the AlN [i.e. either amorphous (a-AlN), or wurtzite (w-AlN)]. The structure of those samples was asserted by TEM/HRTEM observations and XRD measurements. In all cases, Ag was arranged in 21 layers, while the multilayer structure always started and ended with AlN. XTEM images, recorded along the $[1-10]_{\mathrm{Si}}$ zone axis confirmed the multilayer structure of the samples, as illustrated in Fig. 1. The Ag layers comprised of spherical, single phase NPs, which however are abutting on each other; as a result, the as-grown multilayers do not exhibit LSPR. Selected area electron diffraction (SAED) initially confirmed the absence of AlN crystallinity in samples MS1 and MS2. A representative SAED pattern is presented in Fig 1c, showing rings corresponding exclusively to Ag $d$-spacings. Sample MS3 exhibited additional rings originating from the w-AlN structure, as shown in Fig 1e. Further confirmation was obtained by HRTEM. Figs $2 \mathrm{a}$ and $2 \mathrm{~b}$ illustrate NPs with characteristic $\{111\} \mathrm{Ag} d$-spacings surrounded by a-AlN and nanocrystalline w-AIN in samples MS2 and MS3 respectively. In all samples, the formation of a zone of pure amorphous AlN depleted adjacent to Si was observed; the formation of this zone is common to all AlN:Ag nanocomposites independently of the fabrication technique and process and has been observed in Refs. 28-30, as well. In particular, for the case of MS3 this zone extended to $2.5 \mathrm{~nm}$ of thickness on average as shown in detail in Fig 2c, while for samples MS1 and MS2 (Fig $2 \mathrm{a}$ and $\mathrm{b}$ ) it further extended to $6.5 \mathrm{~nm}$ average thickness. Sharp AIN/Si interfaces were observed for samples MS1 and MS2, while for MS3 a slight Si roughening was introduced due to the higher power employed for AlN deposition. The NP diameters were, on average, in the range of 6-8 nm for sample MS1, 4-6 nm for MS2, and 2-4 nm for MS3.

XRD may also provide vital information regarding the crystal structure of the as-grown films. Although XRD lacks the resolution of TEM, it provides structural information of higher statistical significance all over the volume and surface of the sample. Indeed, XRD patterns, which are 
illustrated in Fig. 3, revealed that for samples MS1 and MS2 there is no indication of AlN crystallinity, while the diffraction signal from the $\operatorname{Ag}(111)$ planes is clearly observed. On the contrary, the sample MS3 did not exhibit any diffraction signal associated with Ag, indicating that any Ag NPs are smaller than $4 \mathrm{~nm}$ (XRD amorphous), thus confirming the finer size distribution of Ag nanoparticles identified by TEM for this sample. Regarding the w-AlN phase, which is expected to be formed only in sample MS3 (due to the used deposition conditions [30], and as observed by TEM), XRD provided marginal signals for the (100) and (002) diffraction peaks, which can be hardly resolved from the noise level, confirming the nanocrystalline character of w-AlN.

\subsection{Laser treated samples}

Following deposition, the samples were subjected to LA. The LA process took place in atmospheric pressure (1 Bar) environment and under Ar high-pressure (10 Bar) environment delivering one or two pulses of an $\operatorname{ArF}(193 \mathrm{~nm})$ or a $\mathrm{KrF}(248 \mathrm{~nm})$ laser. At first we performed LA with a single pulse at $193 \mathrm{~nm}$, while the fluence was varying between 400 and $700 \mathrm{~mJ} / \mathrm{cm}^{2}$.

Samples MS2 (a-AlN) and MS3 (w-AlN) were studied by TEM after the LA treatment and were compared in order to ascertain the influence of LA on NP abundance and volume fraction. Figure 4 comprises XTEM images of samples MS2 and MS3 after LA at $600 \mathrm{~mJ} / \mathrm{cm}^{2}$ (one pulse, $193 \mathrm{~nm}$ ) and at 1 and 10 Bar respectively.

Measurements of NP abundance and volume fraction were performed along the whole thickness of the nanocomposite films. In order to obtain accurate statistical results, we measured at least 200 and up to 400 NPs for each sample (the exact number of measured NPs in each case depended on the TEM image). Calculations of the abundance and volume fractions were performed by assuming that the NPs were spherically shaped on average, which is a reasonable approximation, based on our TEM observations. After recording their diameters, the NPs were sorted by size and were divided into classes of $2 \mathrm{~nm}$ range. By counting the number of NPs belonging to each class, we calculated the abundances, according to the equation: 


$$
\text { abundance } \%=\frac{\text { counts per class }}{\text { total counts }} * 100
$$

The volume fractions were obtained from the abundances according to the equation:

$$
\text { volume fraction } \%=\frac{(\text { abundance })_{i} * r_{i}^{3}}{\sum_{i}(\text { abundance })_{i} * r_{i}^{3}}
$$

where $r_{\mathrm{i}}$ denotes the average NP radius for each class.

It can be seen that, in the case of sample MS2 (a-AlN), LA increased the distribution of NPs towards larger diameters. The volume fraction of Ag in large NPs located near the film surface increased significantly. On the other hand, a more uniform distribution of the Ag volume fraction in larger NPs was obtained by LA under high pressure, indicating that the high pressure hinders the outdiffusion of $\mathrm{Ag}$, i.e. the uniform force exerted on the surface suppresses surface modifications during heating of the matrix by LA, and limits the formation of large NPs since Ag atoms must overcome a larger energy barrier. The increase of the volume fraction distribution towards larger NP sizes was more subtle in the case of w-AlN matrix (sample MS3). On the other hand the NP distribution in sample MS3 after high pressure LA was rather similar to that of sample MS2.

After the LA process, the larger Ag NPs emerged close to the sample surface. As shown in our previous contribution [27] there is a significant temperature gradient along the multilayer structure, in the case of a-AlN; higher temperatures are observed close to the surface resulting in an annealed structure with larger NPs towards it, albeit still embedded within the amorphous matrix thus ensuring their protection. Most of the observed NPs were round-shaped, while some of the larger were ellipsoidal. Due to different thermal conductivities of wurtzite and amorphous AlN matrix of the samples, the depth of the affected layers as well as the average size distribution of the NPs varied [27]. In the case of w-AlN (MS3), a constant temperature profile along the film thickness [27] allows for a more uniform NP size distribution. In addition, the non-linearity in thermal conductivity (in relation to the temperature rise within the film) increases the temperature gradient developed in the film, making it sharper as the fluence increases. This means that a composite with 
a matrix of high thermal conductivity (w-AlN, case of MS3) will be restructured only closer to the film's top surface, while a composite with a matrix of low thermal conductivity (a-AlN, cases of MS1 and MS2) will be restructured to a larger extend across the film thickness. This perfectly matches the results from TEM observations shown in Fig. 4. Histograms illustrated in Fig. 5 indicated that, for the case of MS2, the average nanoparticle size was larger than those in MS3. On the other hand, it seems that for both cases the application of high pressure, during LA, suppresses the diffusion of Ag. As a result the formation of smaller NPs $(<4 \mathrm{~nm})$ is favored.

In order to have a more insightful view of the effect of the high pressure during LA and to quantify its effect, we performed detailed XRD analysis. Figure 6 illustrates the XRD patterns of sample MS1, treated by LA under the aforementioned conditions. Based on the fact that no trace of AlN peaks appeared in the diffractograms of LA samples in ambient (pressure 1 Bar, Figs. 6a16a4), it can be confirmed that the AIN matrix remains amorphous after LA in the case of the studied multilayers, in contrast to what was observed in laser-deposited nanocomposite AlN:Ag films $[28,29]$; this is most likely due to the different distribution of the as grown $\mathrm{Ag}$ (in layers in our case and in NPs in Refs. 28,29), which result in different optical absorption and thermal dissipation gradient profiles. On the other hand, well defined Ag (111) peaks appeared in all XRD patterns, which confirmed the existence of crystalline Ag NPs. The observed Ag(111) peaks of LA samples are more prominent than in the case of as-grown samples (Fig. 3) suggesting also an overall increase in NPs' size that confirms the TEM observations. In addition, it is interesting to note that broad $\operatorname{Ag}(200)$ peaks also emerge after LA. In the case of the externally applied high pressure (10, Bar, Figs. 6b1-6b4) during LA, both the $\mathrm{Ag}(111)$ and $\mathrm{Ag}(200)$ exhibit higher signal to noise ratios compared to those of samples laser-annealed at ambient (1 Bar, , Figs. 6a1-6a4). This clearly proves that the application of high pressure during LA hinders, indeed, the outdiffusion and ablation of Ag. In order to quantify the XRD data, the main $\mathrm{Ag}(111)$ peak was fitted with Lorentzian curves and the corresponding broadening (full width at half maximum - FWHM - at $2 \theta$ scale) was used in order to determine the grain size applying Scherrer's equation [34]. The corresponding results for a variety 
of samples and LA conditions are presented in Fig. 7 (note that the statistical error of the grain size values is varying between $0.5-2 \mathrm{~nm}$, therefore the reported values in Fig. 7 demonstrate variations well beyond this error). The main observations include:

i) NP enlargement for successive pulses in all cases, i.e. larger NPs observed for LA with 2 pulses compared to 1 pulse LA, which suggest that the NP enlargement is the result of additive diffusion that is characteristic of an Ostwald ripening process towards the film's surface as indicated by TEM (Fig. 4),

ii) the application of high pressure (10 Bar) during LA does not affect significantly the NP enlargement for the first pulse, where the Ag diffusion is taken place mostly into the film; instead, it results in larger Ag NPs after 2 pulses LA possibly by preventing the Ag ablation from the surface in combination with the successive outdiffusion of $\mathrm{Ag}$ from the film,

iii) there is NP refinement for $700 \mathrm{~mJ} / \mathrm{cm}^{2}$ for all cases; this may define the ablation threshold of Ag from the surface,

iv) larger NPs formed for LA using the $248 \mathrm{~nm}$ instead of the $193 \mathrm{~nm}$ laser for the same sample (Fig. 7b,c); this is exclusively due to the smaller absorption coefficient of AlN for $248 \mathrm{~nm}$ compared to $193 \mathrm{~nm}$ [29] because of AlN's fundamental gap (6.2 eV) [35] resulting in the collaborative effects of overheating the Ag layers [29] and the light penetrating deeper into the film and consequently Ag diffused from a more extended region of the film towards the sample's surface and contributing the Ostwald ripening process,

v) larger NPs formed after LA for thicker Ag layers in the as-grown samples irrespective of the nature of AlN (amorphous or wurtzite) (see Fig. 7a,b,d), which is quite reasonable considering that the available amount of $\mathrm{Ag}$ for diffusion is increasing with Ag layer thickness.

In order to illustrate better the nanometric outdiffusion of Ag during LA and to investigate the effect of the application of high pressure, we performed detailed XPS measurements. XPS spectra from sample MS2 after LA with $248 \mathrm{~nm}$ at ambient and high pressure (10 Bar) are shown in 
Fig. 8a. After the LA process the top surface layers of the sample were enriched with $\mathrm{Ag}$; this is indicated by the corresponding increase of the intensity of the Ag-3d peaks. The analysis shows that the relative percentage of silver in the high pressure treated film's top surface was $\frac{[A g]}{[A l N]} \approx 1.28$, while for the sample processed in atmospheric pressure was $\frac{[A g]}{[A l N]} \approx 2.10$. That confirmed the fact that pressure suppresses Ag diffusion towards the free surface of the film. XPS imaging (Fig. 8b,c) also confirmed the spectroscopic results as the Ag composition contrast between treated (bright region) and untreated (dark region) part of the sample is stronger for the case of the sample processed in atmospheric pressure (Fig. 8b). In addition, the sample processed at high pressure (Fig. 8c) exhibits exceptionally well-defined edges between the treated and untreated areas proving that the application of high pressure inhibits the surface diffusion of Ag, as well.

The diffusion and coarsening of Ag NPs affect severely the plasmonic response of the LA samples. As a demonstration of this effect, we present in Fig. 9 the optical reflectivity spectra of sample MS2 after LA with the $248 \mathrm{~nm}$ laser at various fluences $\left(400-700 \mathrm{~mJ} / \mathrm{cm}^{2}\right.$, black, red, blue and dark cyan lines, respectively), pressures (1 Bar - solid lines, or 10 Bars - dotted lines) and number of pulses (1 pulse at Fig. 9a-left panel, or 2 pulses at Fig. 9b-right panel). Note that sample MS2 is identical with the a-AlN/Ag sample whose optical reflectivity spectra after LA with the 193 nm laser were presented in Ref. 27. Therefore, comparing the spectra of Fig. 9a (solid lines) and the data in Ref. 27 we can also establish a comparison of the effects induced by the different LA wavelength. When the $248 \mathrm{~nm}$ laser is used for LA the resulting LSPR peak is located around 720 nm (Fig. 9a, solid lines), while for the case of $193 \mathrm{~nm}$ LA the LSPR is located around $470 \mathrm{~nm}$ (Ref. 27). Thus, we identify that there is substantial redshift in the LSPR band, when the $248 \mathrm{~nm}$ laser is used for annealing. This confirms the XRD findings that the LA with $248 \mathrm{~nm}$ results in overheating of Ag layers and promotes the diffusion and coarsening of Ag NPs. In addition, in both considered cases (193 or $248 \mathrm{~nm}$ LA using one pulse in ambient pressure) the spectral position of LSPR does not vary substantially with laser fluence, instead, the LSPR strength increases with the laser fluence. 
On the contrary, when the LA process is taking place in high pressure and at low fluence (Fig. 9a, black dotted line) the LSPR band is observed in shorter wavelength $(570 \mathrm{~nm})$, indicating the existence of smaller Ag NPs that are gradually enlarged with laser fluence (LSPR shifts to longer wavelengths beyond $750 \mathrm{~nm}$ - Fig. 9a dark cyan line), in agreement with the XRD observations. The reflectivity spectra after LA with two pulses are also consistent with this description. In particular, the LA in high pressure (Fig. 9b - dotted lines) results in strong LSPR around $650 \mathrm{~nm}$, which indicates the massive coarsening of Ag NPs. For LA in ambient conditions (Fig. 9b - solid lines) the Ag outdiffusion is stronger resulting in a continuous Ag layer that does not exhibit LSPR at all; instead these samples have the optical response of metallic Ag confirming the XPS results. In both cases (1 and $10 \mathrm{Bar}$ ), at $700 \mathrm{~mJ} / \mathrm{cm}^{2}$ reduced reflectivity values are observed that indicate the activation of the ablation process, in agreement with the XRD results.

\section{CONCLUSIONS}

Multilayered AlN:Ag films of various intermediate layer's thicknesses were prepared by dual cathode reactive magnetron sputtering. The untreated samples exhibit well formed multilayered structure, with sharp AlN/Ag interfaces, which however do not exhibit any LSPR. The films' original structure was modified after the LA process. The study of the laser-treated samples revealed the formation of single phase, mostly spherical and well-embedded metallic Ag NPs into the film, which are gradually outdiffusing towards the surface. Their size depended on the annealing conditions, as well as from the available silver's content. In particular, the implementation of LA using the $248 \mathrm{~nm}$ results in stronger diffusion phenomena due to the overheating of the Ag layers. On the other hand, the external applied pressure suppressed the Ag's surface diffusion and outdiffusion. For laser fluences exceeding $600 \mathrm{~mJ} / \mathrm{cm}^{2} \mathrm{Ag}$ ablation starts resulting in less pronounced particle coarsening, compared to fluences below $500 \mathrm{~mJ} / \mathrm{cm}^{2}$, possibly due to the activation of the ablation process. 


\section{ACKNOWLEDGEMENTS}

The research activities that led to these results were co-financed by Hellenic Funds and by the European Regional Development Fund (ERDF) under the Hellenic National Strategic Reference Framework (NSRF) 2007-2013, according to contract no. $11 \Sigma \mathrm{YN}-5-1280$ of the Project 'NanoHybrid', within the Program 'Competitiveness and Entrepreneurship'. 


\section{REFERENCES}

1. L. Maya, W.R. Allen, A.L. Glover, J.C. Mabon, Gold nanocomposites, J. Vac. Sci. Technol. B13 (1995) 361-365.

2. J.F. Pierson, D. Wiederkehr, A. Billard, Reactive magnetron sputtering of copper, silver, and gold, Thin Solid Films 478 (2005) 196-205.

3. G. Matenoglou, G.A. Evangelakis, C. Kosmidis, S. Foulias, D. Papadimitriou, P. Patsalas, Pulsed laser deposition of amorphous carbon/silver nanocomposites, Appl. Surf. Sci. 253 (2007) 8155-8159.

4. J.L. Endrino, R. Escobar Galindo, H.-S. Zhang, M. Allen, R. Gago, A. Espinosa, A. Anders, Structure and properties of silver-containing a-C(H) films deposited by plasma immersion ion implantation, Surf. Coat. Technol. 202 (2008) 3675-3682.

5. Y. Wei, R. Klajn, A.O. Pinchuk, B.A. Grzybowski, Synthesis, shape control, and optical properties of hybrid $\mathrm{Au} / \mathrm{Fe}_{3} \mathrm{O}_{4}$ nanoflowers, Small 4 (2008) 1635-1639.

6. J.L. Endrino, D. Horwat, R. Gago, J. Andersson, Y.S. Liu, J. Guo, A. Anders, Electronic structure and conductivity of nanocomposite metal $(\mathrm{Au}, \mathrm{Ag}, \mathrm{Cu}, \mathrm{Mo})$-containing amorphous carbon films, Solid State Sci. 11 (2009) 1742-1746.

7. A.A. Voevodin, J.J. Hu, J.G. Jones, T.A. Fitz, J.S. Zabinski, Growth and structural characterization of yttria-stabilized zirconia-gold nanocomposite films with improved toughness, Thin Solid Films 401 (2001) 187-195.

8. A. Lotsari, G.P. Dimitrakopulos, Th. Kehagias, P. Kavouras, H. Zoubos, L.E. Koutsokeras, P. Patsalas, Ph. Komninou, Structure, stability and mechanical performance of AlN:Ag nanocomposite films, Surf. Coat. Technol. 204 (2010) 1937-1941.

9. N.M. Figueiredo, F. Vaz, L. Cunha, S.E. Rodil, A. Cavaleiro, Structural, chemical, optical and mechanical properties of Au doped AlN sputtered coatings, Surf. Coat. Technol. 255 (2014) 130-139. 
10. H. Li, Z. Bian, J. Zhu, Y. Huo, H. Li, Y. Lu, Mesoporous $\mathrm{Au} / \mathrm{TiO}_{2}$ nanocomposites with enhanced photocatalytic activity, J. Amer. Chem. Soc. 129 (2007) 4538-4539.

11. D. Horwat, J.L. Endrino, A. Boreave, R. Karoum, J.F. Pierson, S. Weber, A. Anders, Ph. Vernoux, Deep oxidation of methane on particles derived from YSZ-supported Pd-Pt-(O) coatings synthesized by Pulsed Filtered Cathodic Arc, Catal. Commun.10 (2009) 1410-1413.

12. N. Udawatte, M. Lee, J. Kim, D. Lee, Well-defined Au/ZnO nanoparticle composites exhibiting enhanced photocatalytic activities, ACS Appl. Mater. Interf. 3 (2011) 4531-4538.

13. S. Verma, A.J. Domb, N. Kumar, Nanomaterials for regenerative medicine, Nanomedicine 6 (2011) 157-181.

14. M. Andara, A. Agarwal, D. Scholvin, R.A. Gerhardt, A. Doraiswamy, C. Jin, R.J. Narayan, C.C. Shih, C.-M. Shih, S.-J. Lin, Y.-Y. Su, Hemocompatibility of diamondlike carbon-metal composite thin films, Diam. Relat. Mater. 15 (2006) 1941-1948.

15. R.J. Narayan, H. Abernathy, L. Riester, C.J. Berry, R. Brigmon, Antimicrobial properties of diamond-like carbon-silver-platinum nanocomposite thin films, J. Mater. Eng. Perfor. 14 (2005) 435-440.

16. J.L. Endrino, J.C. Sánchez-López, R.E. Galindo, D. Horwat, A. Anders, Beneficial silver: Antibacterial nanocomposite Ag-DLC coating to reduce osteolysis of orthopaedic implants, J. Phys. Conf. Ser. 252 (2010) art. no12005.

17. C. Lopes, P. Fonseca, T. Matamá, A. Gomes, C. Louro, S. Paiva, F. Vaz, Protective Ag:TiO 2 thin films for pressure sensors in orthopedic prosthesis: The importance of composition, structural and morphological features on the biological response of the coatings, J. Mater. Sci. 25 (2014) 2069-2081.

18. J. García-Serrano, A.G. Galindo, U. Pal, $\mathrm{Au}-\mathrm{Al}_{2} \mathrm{O}_{3}$ nanocomposites: XPS and FTIR spectroscopic studies, Sol. Ener. Mater. Sol. Cells 82 (2004) 291-298. 
19. S. Muduli, O. Game, V. Dhas, K. Vijayamohanan, K.A. Bogle, N. Valanoor, S.B. Ogale, TiÖAu plasmonic nanocomposite for enhanced dye-sensitized solar cell (DSSC) performance, Sol. Ener. 86 (2012) 1428-1434.

20. H. Zoubos, L.E. Koutsokeras, D.F. Anagnostopoulos, E. Lidorikis, S.A. Kalogirou, A.R. Wildes, P.C. Kelires, P. Patsalas, Broadband optical absorption of amorphous carbon/Ag nanocomposite films and its potential for solar harvesting applications, Sol. Ener. Mater. Sol. Cells 117 (2013) 350-356.

21. E. Pedrueza, J.L. Valdés, V. Chirvony, R. Abargues, J. Hernández-Saz, M. Herrera, S.I. Molina, J.P. Martínez-Pastor, Novel method of preparation of gold-nanoparticle-doped $\mathrm{TiO}_{2}$ and $\mathrm{SiO}_{2}$ plasmonic thin films: Optical characterization and comparison with Maxwell-Garnett modeling, Adv. Funct. Mater. 21 (2011) 3502-3507.

22. N.A. Joy, M.I. Nandasiri, P.H. Rogers, W. Jiang, T. Varga, S.V.N.T. Kuchibhatla, S. Thevuthasan, M.A. Carpenter, Selective plasmonic gas sensing: $\mathrm{H}_{2}, \mathrm{NO}_{2}$, and $\mathrm{CO}$ spectral discrimination by a single $\mathrm{Au}-\mathrm{CeO}_{2}$ nanocomposite film, Anal. Chem. 84 (2012) 5025-5034.

23. J. Toudert, L. Simonot, S. Camelio, D. Babonneau, Advanced optical effective medium modeling for a single layer of polydisperse ellipsoidal nanoparticles embedded in a homogeneous dielectric medium: Surface plasmon resonances, Phys. Rev. B86 (2012) art. no. 45415.

24. J. Toudert, S. Camelio, D. Babonneau, M.-F. Denanot, T. Girardeau, J.P. Espiños, F. Yubero, A.R. Gonzalez-Elipe, Morphology and surface-plasmon resonance of silver nanoparticles sandwiched between $\mathrm{Si}_{3} \mathrm{~N}_{4}$ and BN layers, J. Appl. Phys. 98 (2005) art. no. 114316.

25. I. Moura, M.F. Cerqueira, D. Melnikau, D. Savateeva, Y. Rakovich, J. Borges, F. Vaz, M. Vasilevskiy, Effect of surface plasmon resonance in $\mathrm{TiO}_{2} / \mathrm{Au}$ thin films on the fluorescence of self-assembled CdTe QDs structure, J. Phys. Conf. Ser. 605 (2015) art. no. 12025. 
26. M. Torrell, R. Kabir, L. Cunha, M.I. Vasilevskiy, F. Vaz, A. Cavaleiro, E. Alves, N.P. Barradas, Tuning of the surface plasmon resonance in $\mathrm{TiO} 2 / \mathrm{Au}$ thin films grown by magnetron sputtering: The effect of thermal annealing, J. Appl. Phys. 109 (2011) art. no. 74310.

27. A. Siozios, N. Kalfagiannis, D.V. Bellas, C. Bazioti, G.P. Dimitrakopulos, G. Vourlias, W.M. Cranton, E. Lidorikis, D.C. Koutsogeorgis, P. Patsalas, Sub-surface laser nanostructuring in stratified metal/dielectric media: A versatile platform towards flexible, durable and large-scale plasmonic writing, Nanotechnology 26 (2015) art. no. 155301.

28. A. Siozios, D.C. Koutsogeorgis, E. Lidorikis, G.P. Dimitrakopulos, T. Kehagias, H. Zoubos, Ph. Komninou, W.M. Cranton, C. Kosmidis, P. Patsalas, Optical encoding by plasmon-based patterning: Hard and inorganic materials become photosensitive, Nano Lett. 12 (2012) 259-263.

29. A. Siozios, D.C. Koutsogeorgis, E. Lidorikis, G.P. Dimitrakopulos, N. Pliatsikas, G. Vourlias, T. Kehagias, P. Komninou, W.M. Cranton, C. Kosmidis, P. Patsalas, Laser-matter interactions, phase changes and diffusion phenomena during laser annealing of plasmonic AlN:Ag templates and their applications in optical encoding, J. Phys. D48 (2015) art. no. 285306.

30. A. Siozios, H. Zoubos, N. Pliatsikas, D.C. Koutsogeorgis, G. Vourlias, E. Pavlidou, W.M. Cranton, P. Patsalas, Growth and annealing strategies to control the microstructure of AlN: Ag nanocomposite films for plasmonic applications, Surf. Coat. Technol. 255 (2014) 28-36.

31. M. Torrell, P. Machado, L. Cunha, N.M. Figueiredo, J.C. Oliveira, C. Louro, F. Vaz, Development of new decorative coatings based on gold nanoparticles dispersed in an amorphous $\mathrm{TiO}_{2}$ dielectric matrix, Surf. Coat. Technol. 204 (2010) 1569-1575.

32. S. Camelio, J. Toudert, D. Babonneau, T. Girardeau, Tailoring of the optical properties of Ag: $\mathrm{Si}_{3} \mathrm{~N}_{4}$ nanocermets by changes of the cluster morphology, Appl. Phys. B80 (2005) 89-96.

33. S. Camelio, D. Babonneau, T. Girardeau, J. Toudert, F. Lignou, M.-F. Denanot, N. Maître, A. Barranco, P. Guérin, Optical and structural properties of $\mathrm{Ag}-\mathrm{Si}_{3} \mathrm{~N}_{4}$ nanocermets prepared by means of ion-beam sputtering in alternate and codeposition modes, Appl. Opt. 42 (2003) 674681. 
34. A.L. Patterson, The Scherrer formula or X-ray particle size determination, Phys. Rev. 56 (1939) 978-982.

35. Ch.E. Lekka, P. Patsalas, Ph. Komninou, G.A. Evangelakis, Electronic properties and bonding characteristics of AlN:Ag thin film nanocomposites, J. Appl. Phys. 109 (2009) art. no. 054310. 
Table 1. Summary of growth conditions and structural properties of the samples

\begin{tabular}{cccccccc}
\hline Sample & Al & Ag & AlN layer & AlN layer & Ag layer & AlN nominal & Ag nominal \\
& Power & Power & crystalline & deposition & deposition & thickness & thickness \\
& (Watt) & (Watt) & state & time (sec) & time (sec) & $(\mathrm{nm})$ & $(\mathrm{nm})$ \\
MS1 & 15 & 10 & Amorphous & 1028 & 30 & 10 & 5 \\
MS2 & 15 & 10 & Amorphous & 1200 & 10 & 12 & 3 \\
MS3 & 100 & 10 & Wurtzite & 60 & 10 & 14 & 1 \\
\hline
\end{tabular}




\section{FIGURE CAPTIONS}

Figure 1. (a) (b), and (c) Bright field XTEM images obtained along the [1-10] $]_{\text {Si }}$ zone axis of untreated Ag:AlN samples MS1, MS2 with a-AlN matrix, and MS3 with w-AlN matrix respectively.

(d) Representative composite SAED pattern from sample MS2 showing rings corresponding to Ag d-spacings. (e) SAED pattern from sample MS3 exhibiting Ag and w-AlN rings.

Figure 2. (a) and (b) HRTEM images of NPs in samples MS2 and MS3 respectively, depicting the surrounding amorphous and nanocrystalline surrounding AlN matrix in each respective case. (c) HRTEM image of the interfacial region in sample MS3 showing an amorphous AlN zone depleted of $\mathrm{Ag}$ and small roughening of the Si substrate.

Figure 3. Grazing Incidence XRD patterns of the three as-grown AlN/Ag multilayers considered in this study.

Figure 4. (a) and (b) Bright field XTEM images of sample MS2 (a-AIN), after ambient and high pressure LA at $600 \mathrm{~mJ} / \mathrm{cm}^{2}$,respectively, (c) and (d) Similar images for sample MS3 (w-AlN).

Figure 5. Histograms of NP abundance and volume fraction vs NP diameter for sample MS2 (a) untreated, (b) annealed at ambient, and (c) annealed at high pressure at $600 \mathrm{~mJ} / \mathrm{cm}^{2}$. (d), (e) and (f) Similar histograms for sample MS3 (w-AlN).

Figure 6. Grazing Incidence XRD patterns of MS1 by LA (one pulse of $193 \mathrm{~nm}$ ). Left (a1-a4): in ambient (1 Bar), and right (b1-b4) at Ar high pressure (10 Bar). In both sides, from top to bottom diffractograms correspond to fluence of 400, 500, 600 and $700 \mathrm{~mJ} / \mathrm{cm}^{2}$.

Figure 7. The evolution of average grain size determined by Scherrer's analysis $v s$. the laser fluence for various samples and LA conditions.

Figure 8. (a) XPS spectra from sample MS2: as-grown (black line), after LA with one pulse of 248 $\mathrm{nm}$ in ambient (green line) and under high pressure (wine line). XPS imaging for Ag-3d peak of two 
laser annealed spots on sample MS2: (b) in ambient pressure, and (c) in high pressure. Dark and light region correspond to untreated and laser annealed areas of the sample's surface, respectively.

Figure 9. Optical reflectivity spectra at normal incidence for the sample MS2 after LA with $248 \mathrm{~nm}$ laser at various fluences and pressures for (a) one pulse, and (b) two pulses. 


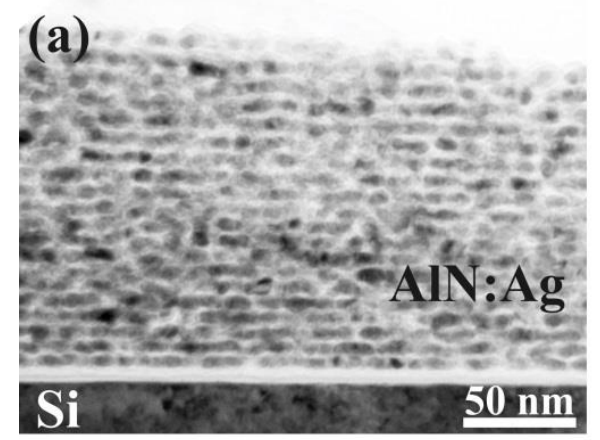

(b)

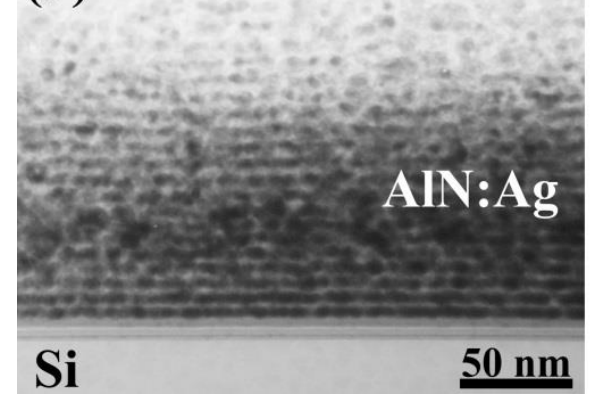

(c)

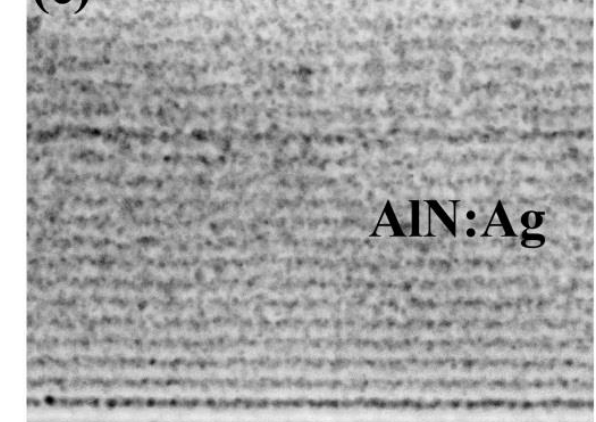

$\mathrm{Si} \quad 50 \mathrm{~mm}$ (d)

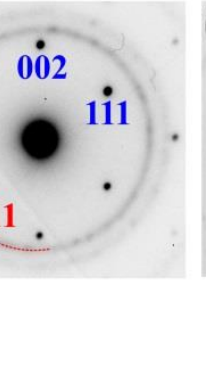

(e)

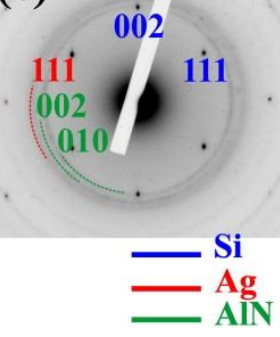

Figure 1 

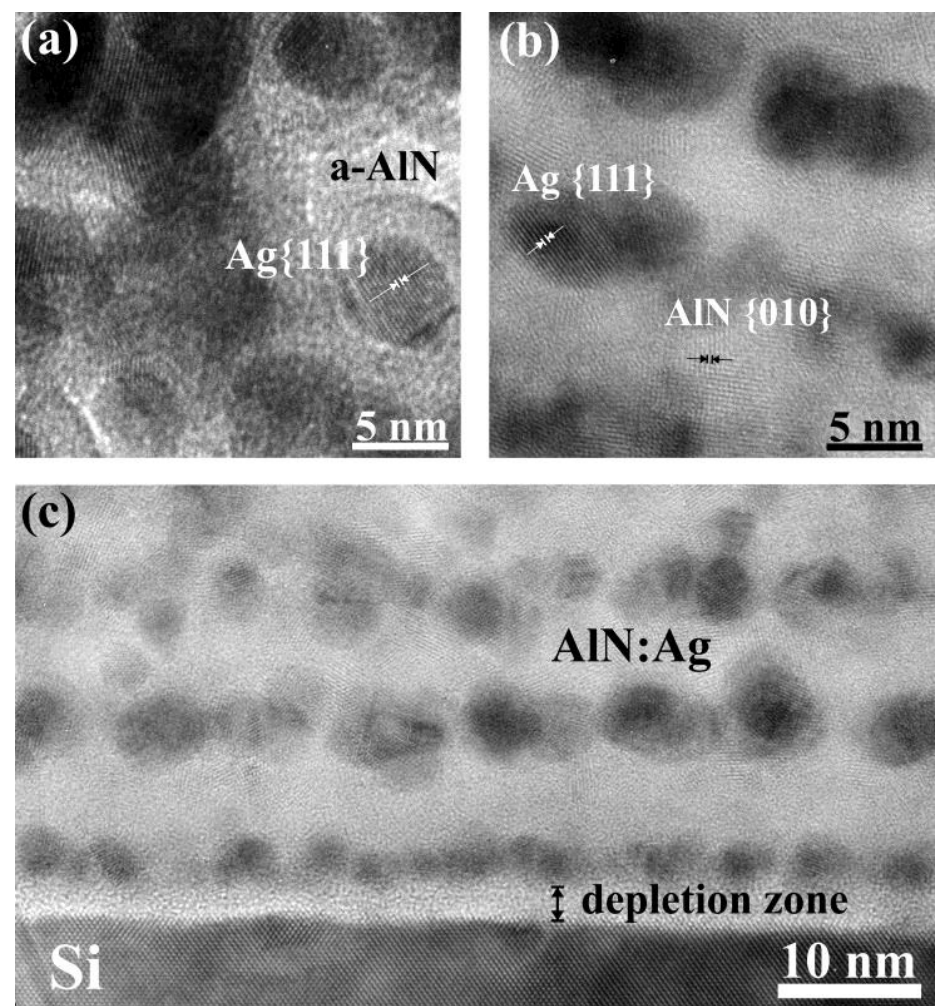

Figure 2 


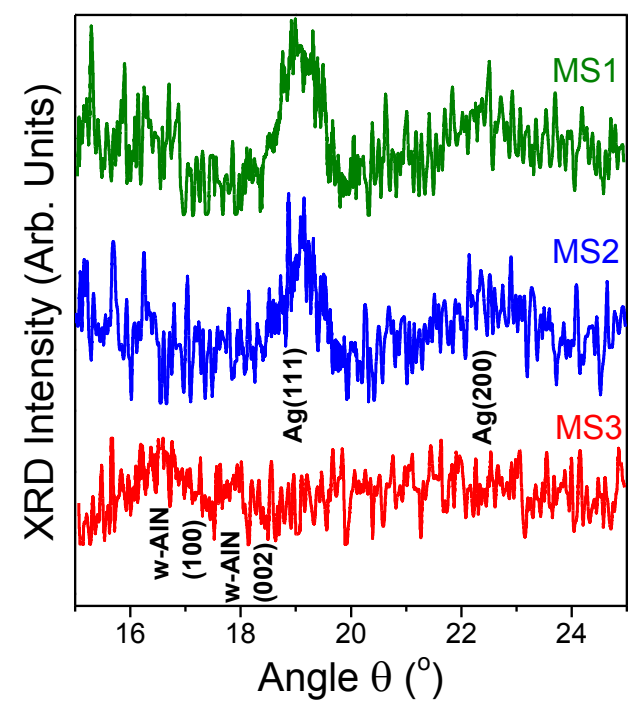

Figure 3 

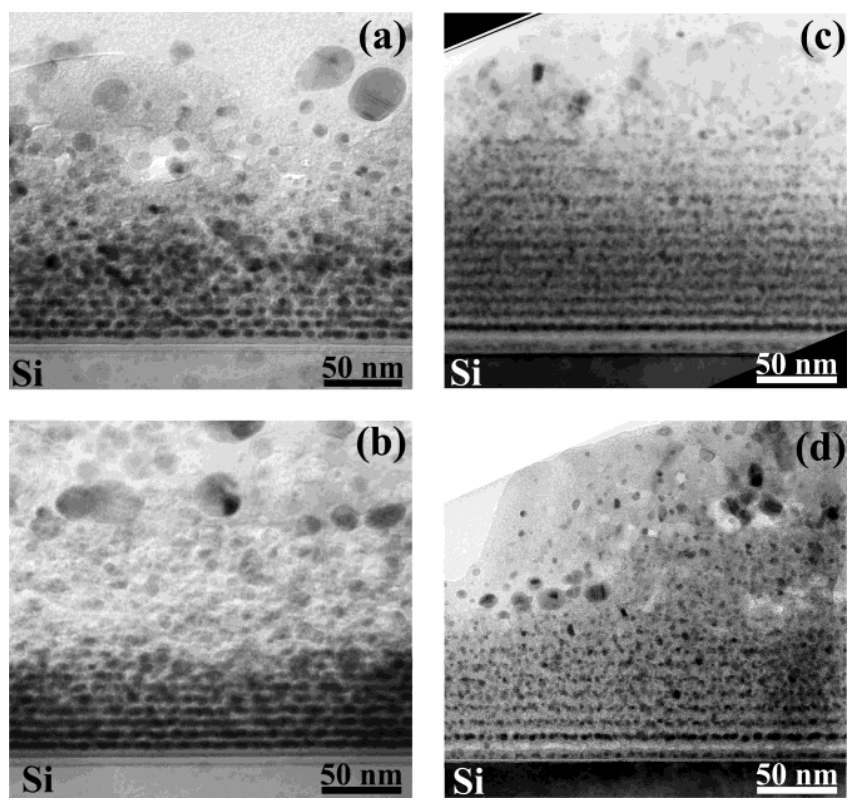

Figure 4 
(a)

(d)
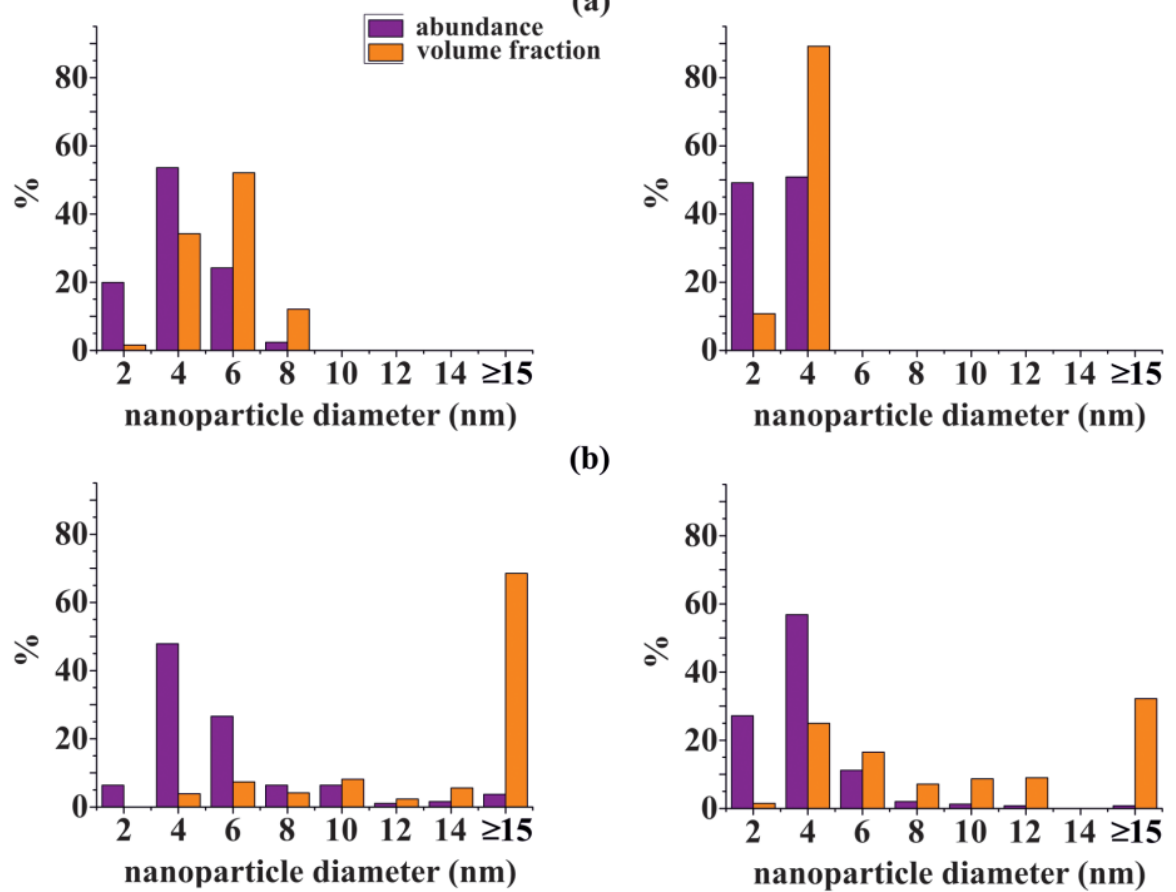

(b)
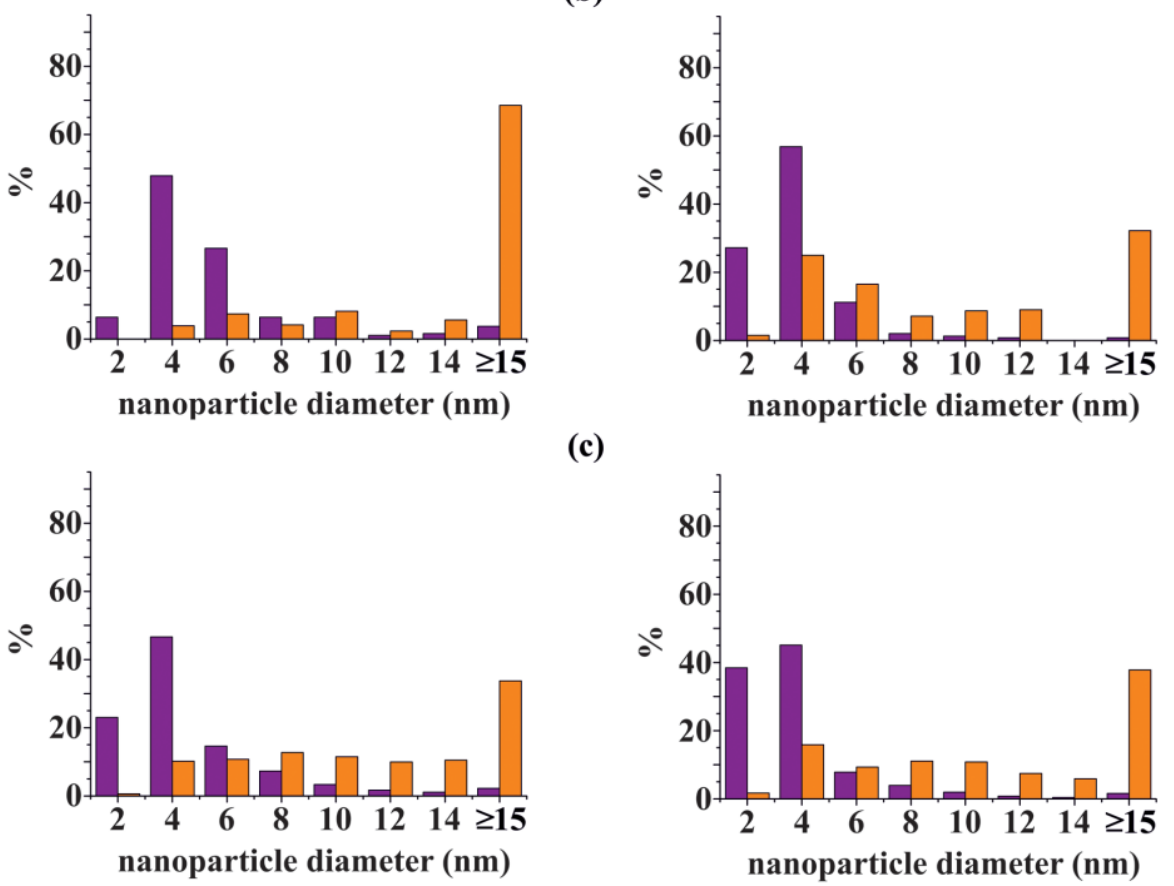

(c)

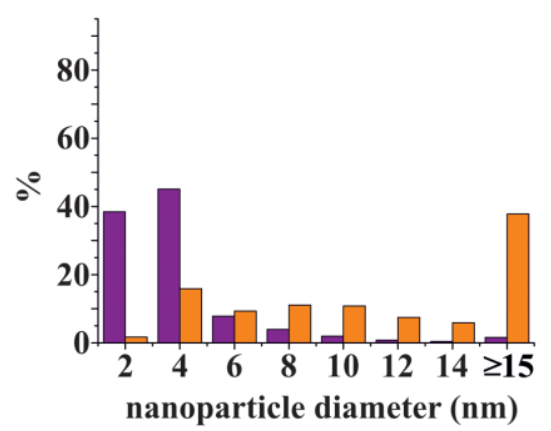

Figure 5 


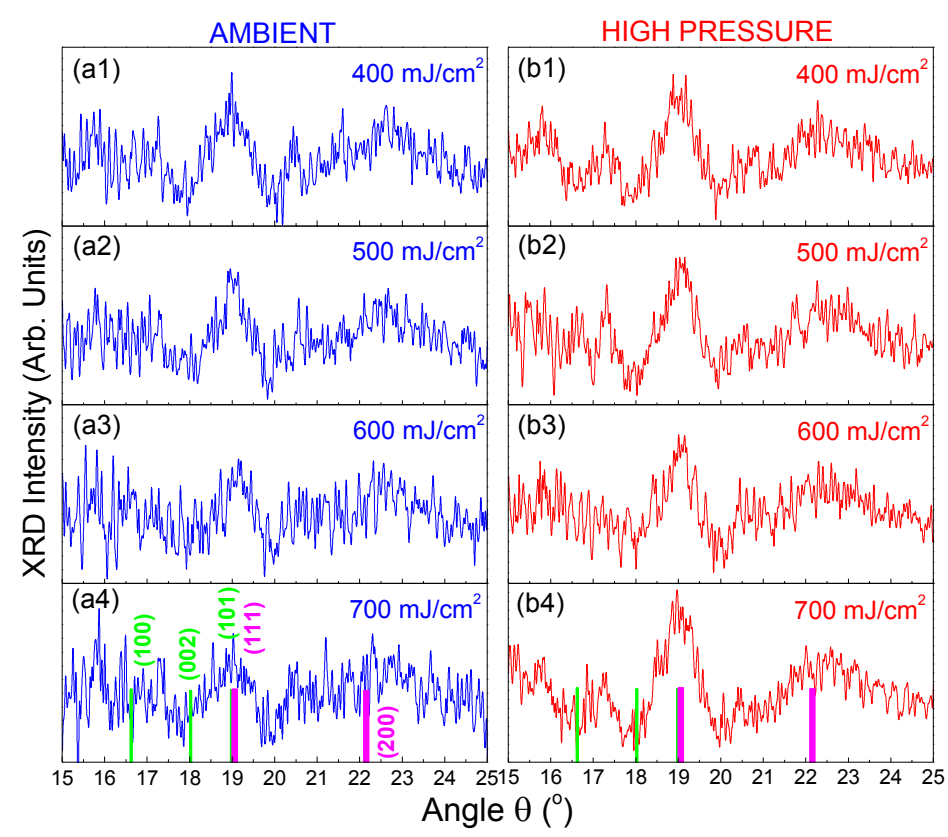

Figure 6 


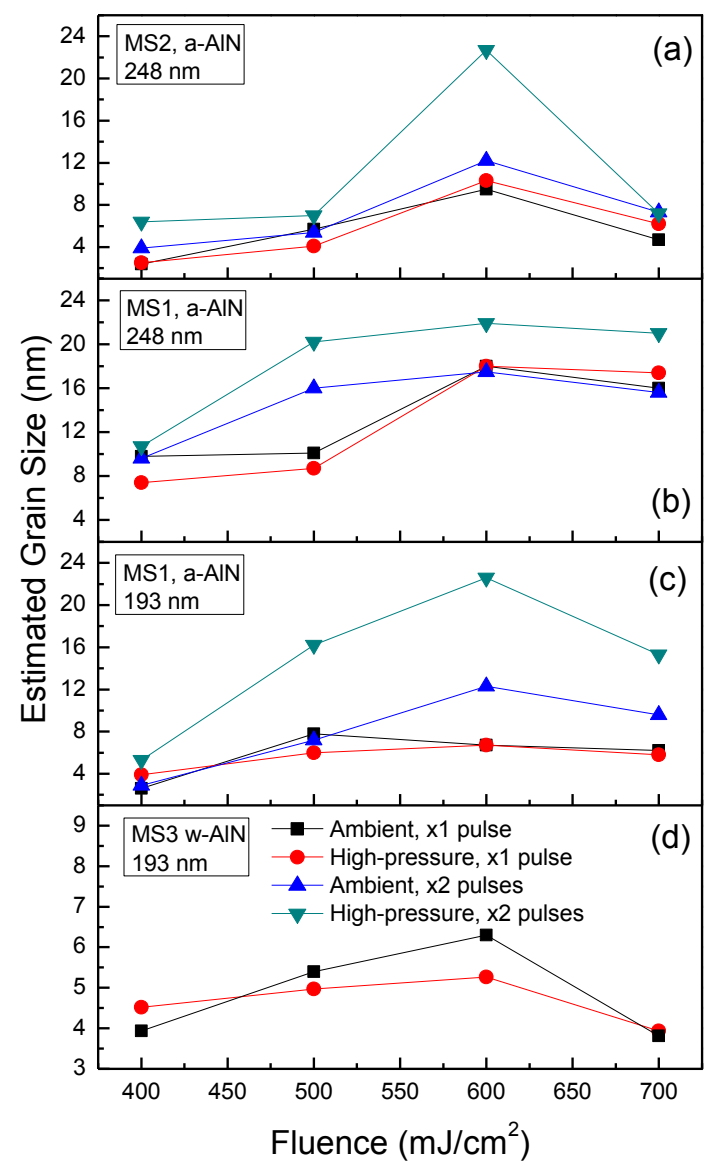

Figure 7 


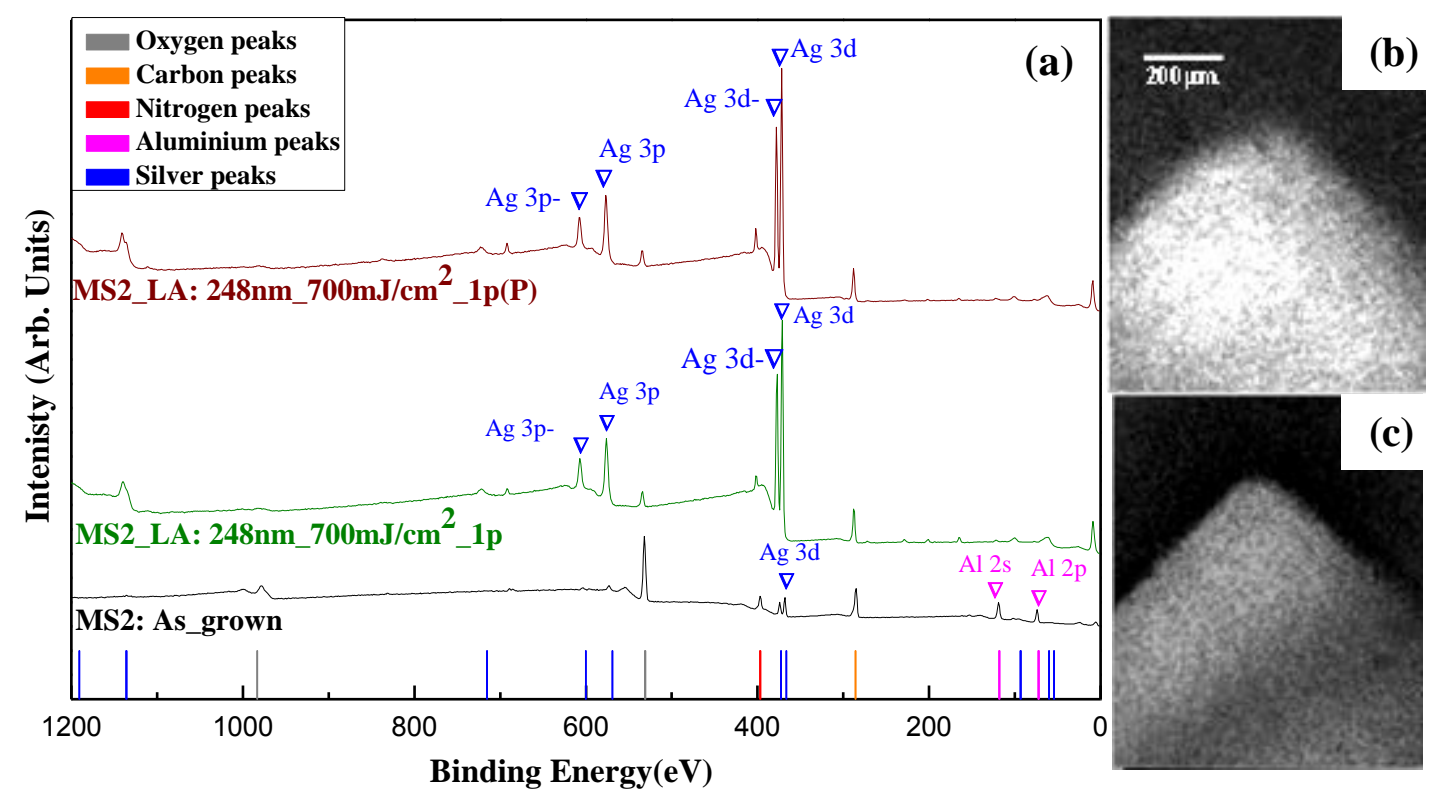

Figure 8 

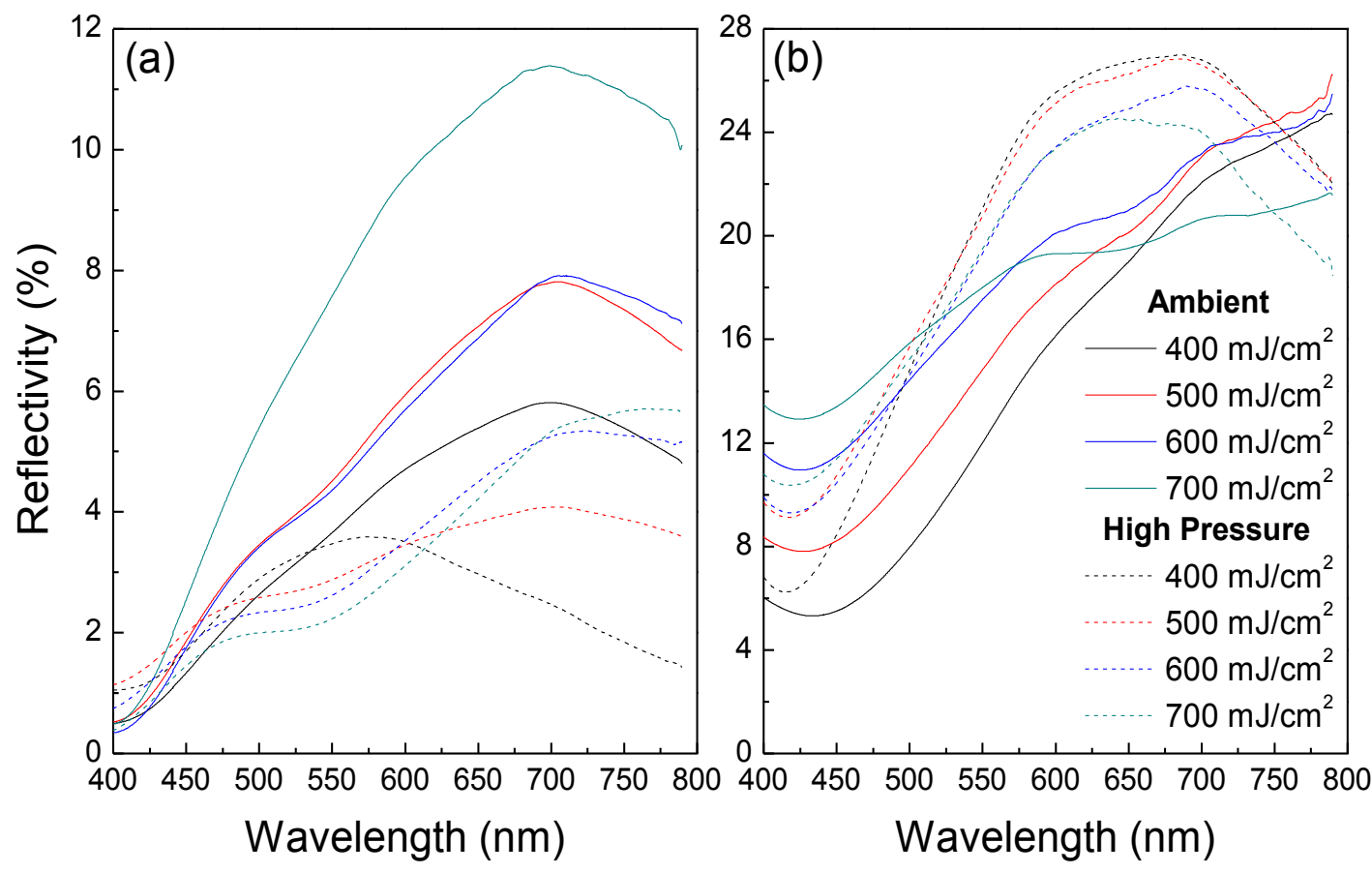

Figure 9 